\title{
A Multichannel IR Sensor System for Condition Monitoring of Technical Fluids
}

\author{
Torsten Bley ${ }^{1}$, Andreas Schütze ${ }^{1,2}$ \\ ${ }^{1}$ Zentrum für Mechatronik und Automatisierungstechnik $\mathrm{GmbH}$, \\ Gewerbepark Eschbergerweg, 66125 Saarbrücken \\ ${ }^{2}$ Lehrstuhl für Messtechnik, Universität des Saarlandes, Saarbrücken
}

\begin{abstract}
In this paper we report about the first results of a novel multichannel oil sensor system, which is able to analyze the deterioration of technical fluids in continuous action. The sensor system is based on a miniaturized measurement cell with a commercial infrared light source and four thermopile detectors with different narrow bandwidth filters. The goal is to provide a small sensor system to use the full lifetime of the technical fluids without any danger of damaging the machine or hydraulic system. The main approach to achieve this goal is to develop a system able to measure the changes in the infrared spectrum of many technical fluids. Therefore the characteristic wavelength of the spectral bands of the technical fluids which are representative for deterioration were analyzed and determined to configure a thermopile sensor with corresponding narrow band filters. The functionality was tested using artificially aged hydraulic fluids and aged oil taken from a combined heat and power unit (CHP). The paper describes the set-up of the sensor system and shows results of characterization tests and first test cycles with mineral and synthetic oils.
\end{abstract}

\section{Motivation}

The properties of technical fluids are strongly dependent on their actual operating characteristics. This makes a prediction of the remaining lifetime of the fluids very difficult for the operating company. Therefore the determination of the current fluid condition is of interest in many applications making use of technical fluids. In order to avoid machine downtime due to excessive deterioration, oil changes are often made well before reaching a critical state based on a fixed number of operating hours or, alternatively, analyzing oil samples in the lab. However, oil sample analysis is rather costly and some uncertainty concerning the machine condition remains during and between sampling. This problem is especially relevant in applications where complex systems depend on the correct function of a critical component, i.e. a transformer.

With the focus on saving cost through better resource management and the reduction of machine downtimes, exact condition monitoring of technical fluids is becoming increasingly important. In addition to the economic advantages a reduction of the environmental impact of technical systems is highly desirable. Considering the large range of different kinds of technical fluids on the market, there are also differences between optical analyses of the deterioration. To cover a broad application range with a single system requires the ability to measure different characteristics simultaneously and the possibility for simple adaptation of the system to different measurement situations.

\section{Experimental methods}

The measurement system is assembled in a small metallic cube with an inlet and outlet for the technical fluid. In the experimental set-up the fluid is pumped continuously through the measurement system, Fig 1. The basic set-up contains a hydraulic pump, the control module, the sensor module, the data acquisition and a LabVIEW (National Instruments, NI) user-interface for controlling the system and data acquisition. Other methods for determining the oil deterioration were published, e.g. measurement with the attenuated reflection method from K. Kudlaty [1] or chemical corrosive sensors from Ch. Schneidhofer [2] et al. The method used for determination of the deterioration of a technical fluid in this paper is the NDIR principle (Non Dispersive InfraRed, [3]) using transmission measurements to provide a good SNR with a durable system. The required small measurement gap is realized by two silicon plates which are spaced by a thin ceramic layer. The average thickness of this gap is approx. $0.2 \mathrm{~mm}$. The spectral bandwidth of the silicon measurement cell provides a broad measurement window to analyze different oil types, e.g. mineral or synthetic oils. A similar method based on a sapphire measurement cell was used by Sumit et al. to determine the oxidation and water content for phosphate hydraulic esters [4]. Agoston et al. used a flow cell with calcium fluoride windows to determine oxidation of engine oils [5]. 


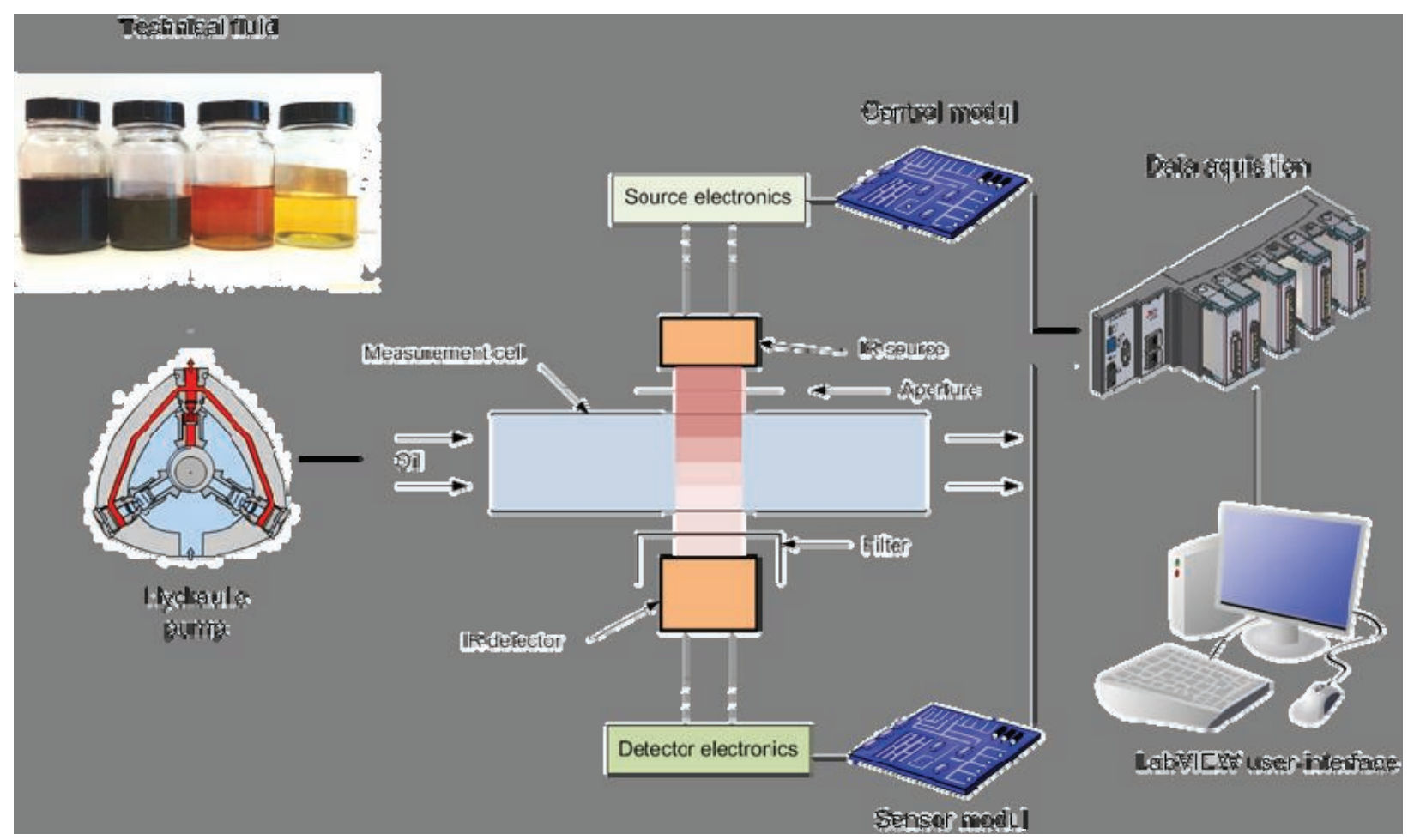

Fig. 1: Measurement set-up consisting of the measurement system, sensor electronics and LabVIEW-User Interface. The Sensor system is tested with continuous fluid flow with regulated pressure, flow rate and temperature to test cross sensitivities.

The used silicon measurement cell in this sensor system is produced by TU IImenau by ion etching, lamination and a pressure assisted sinter process [6]. The small fluid film in the measurement cell is irradiated by a commercial microstructured infrared emitter [7]. The choice of the emitter was based on the almost ideal blackbody behavior providing a broad infrared spectrum as shown in Fig. 2.

The transmitted infrared radiation is measured with a quadruple thermopile, with three thermopiles being used as measurement sensor for condition monitoring and one thermopile as reference to provide an independent measurement of IR source intensity as well as broad band influence like soot. Each thermopile is equipped with a separate narrow bandwidth filter adapted to a relevant part of the received infrared spectrum. The infrared source is powered by a control module to convert the control signals of the data acquisition system into an adapted voltage. In addition, the provided power signal decreases aging effects, e.g. due to electro migration in the infrared source. The sensor module uses an active filter set for the thermopiles to provide an adequate sense voltage. A real-time data acquisition system is used to control the source and to acquire the signals from the four thermopile sensor and also the signals from

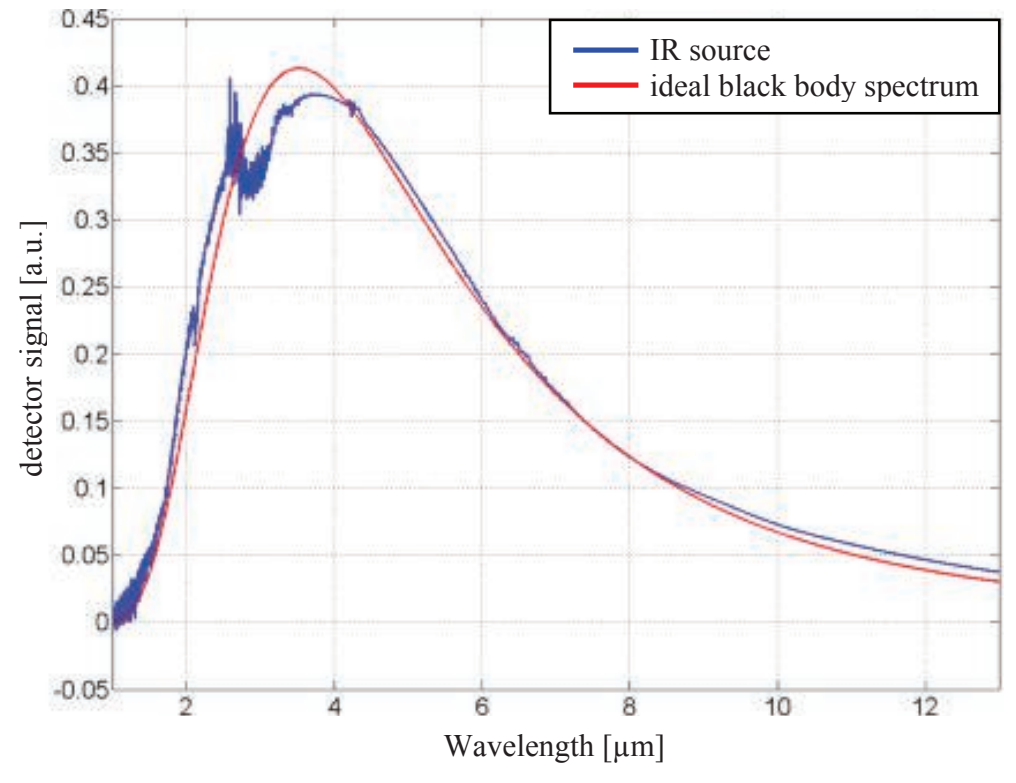
sensors of the hydraulic system. The data acquisition system is connected via ethernet to a PC with a graphical user-interface based on LabVIEW for signal evaluation and interpretation.

To determine the fluid deterioration a rectangle power signal is used to provide simple control electronics, e.g. for mobile solutions. Based on the thermopile time constant the modulation frequency of the infrared source was set to $0.1 \mathrm{~Hz}$.

Fig. 2: Infrared spectrum of the used commercial infrared source. The spectrum was analyzed using an FTIR spectrometer compared with a blackbody reference. The behavior around $2.8 \mu \mathrm{m}$ is caused by water vapor. 
The implemented evaluation software splits the acquired sensor signal into parts with high (IR source on) and low excitation (source off). Using a histogram analysis the average values of the high and low level are determined by minimizing the influence from noise. Based on the high and low level signals the amplitude of the sensor output is calculated to determine the deterioration. For experimental measurements of the oil deteroration different samples of mineral and native oils were aged. This artifcial aging was realized using an aluminum cylinder on a hot plate with magnetic stirrer. At a temperature of $120^{\circ} \mathrm{C}$ the oils were stirred under air bubbling. This method mainly causes oxidation of the oil. To measure different types of aged fluids and to avoid waring of the fluid in the sensor cell the

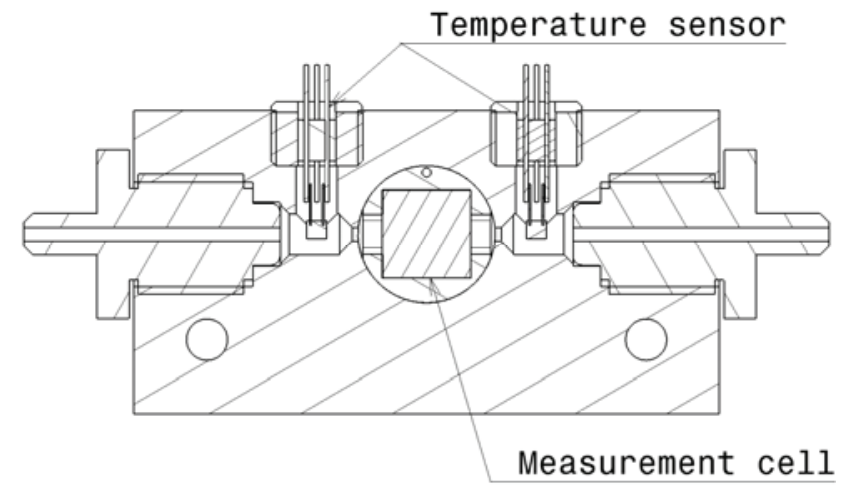

Fig. 3: Compact Sensor System in metallic housing with commercial infrared emitter and quadruple thermopile. sensor system is continously pumped with the fluid. The measurements cell was cleaned between measurements to avoid carry over effects.

To analyze cross sensitivities of the sensor system to the hydraulic conditions the experimental set-up is expandable with a pump with variable flow, a pressure regulating valve and a heating cartridge to control the fluid temperature. To provide a continuous stable flow and pressure environment the real-time system controls the hydraulic pump and a pressure valve by a closed loop control.

\section{Results}

For testing the sensor system native and mineral oils were artificially aged in stages of 3,7 and 10 days for the native oil and 1,6 and 11 days for the mineral oil. A Bruker Vertex 22 FTIR with a $0.2 \mathrm{~mm}$ gap flow cell was used to analyze the infrared spectra of the aged oil samples.

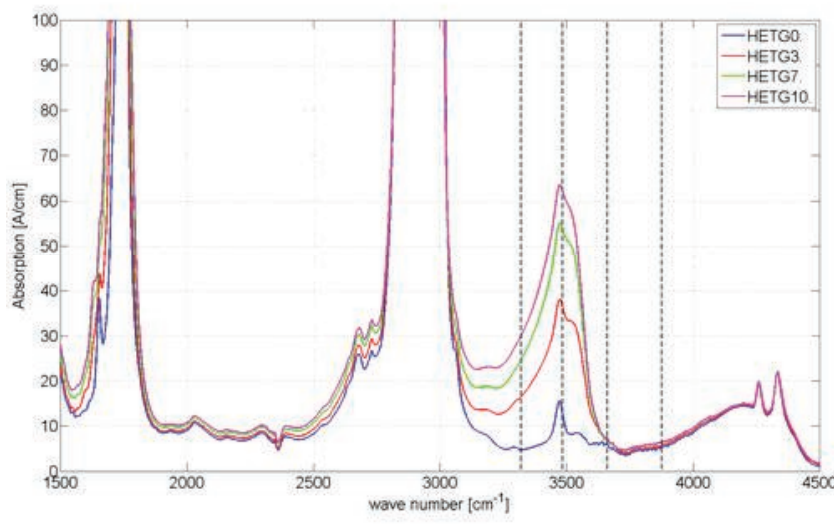

Fig. 4: Optical spectra for aged native hydraulic fluid (Fuchs HETG), including optical filter lines for evaluation in the sensor system.

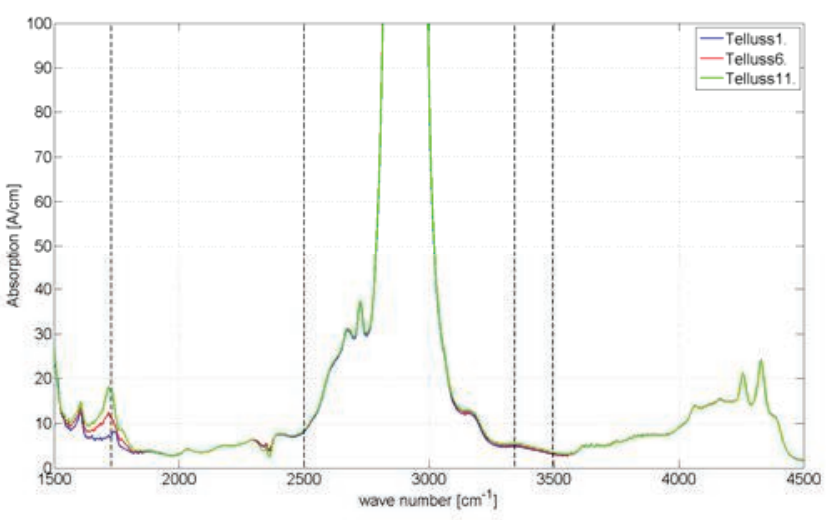

Fig. 5: Optical spectra for aged mineral hydraulic fluid (Shell Telluss), including optical filter lines for evaluation in the sensor system.

The native hydraulic fluid shows a strong increase of the absorption from the new hydraulic fluid to the aged fluids in the infrared spectra in the wave number range from 3100 to $3700 \mathrm{~cm}^{-1}$, as shown in Fig 4 . This spectral characteristic is attributed to the first and second ester base stock breakdown in the standard pratice of condition monitoring from ASTM [8]. The cause for this absorption according to Naldu et al. [9] is the IR characteristic of hydroxyl groups [-OH]. In addition, over the complete spectrum a small increase of the absorption can be observed due to increasing deterioration of the fluid. This is caused by the increasing slush fraction through aging of the fluid. The absorption value for increasing deterioration shows that the absorption difference between the particular aged fluids decreases. For monitoring water and oxidation contents of native hydraulic fluid the optical filter set of the sensor system was specified to $3875,3660,3480$ and $3320 \mathrm{~cm}^{-1}$. 

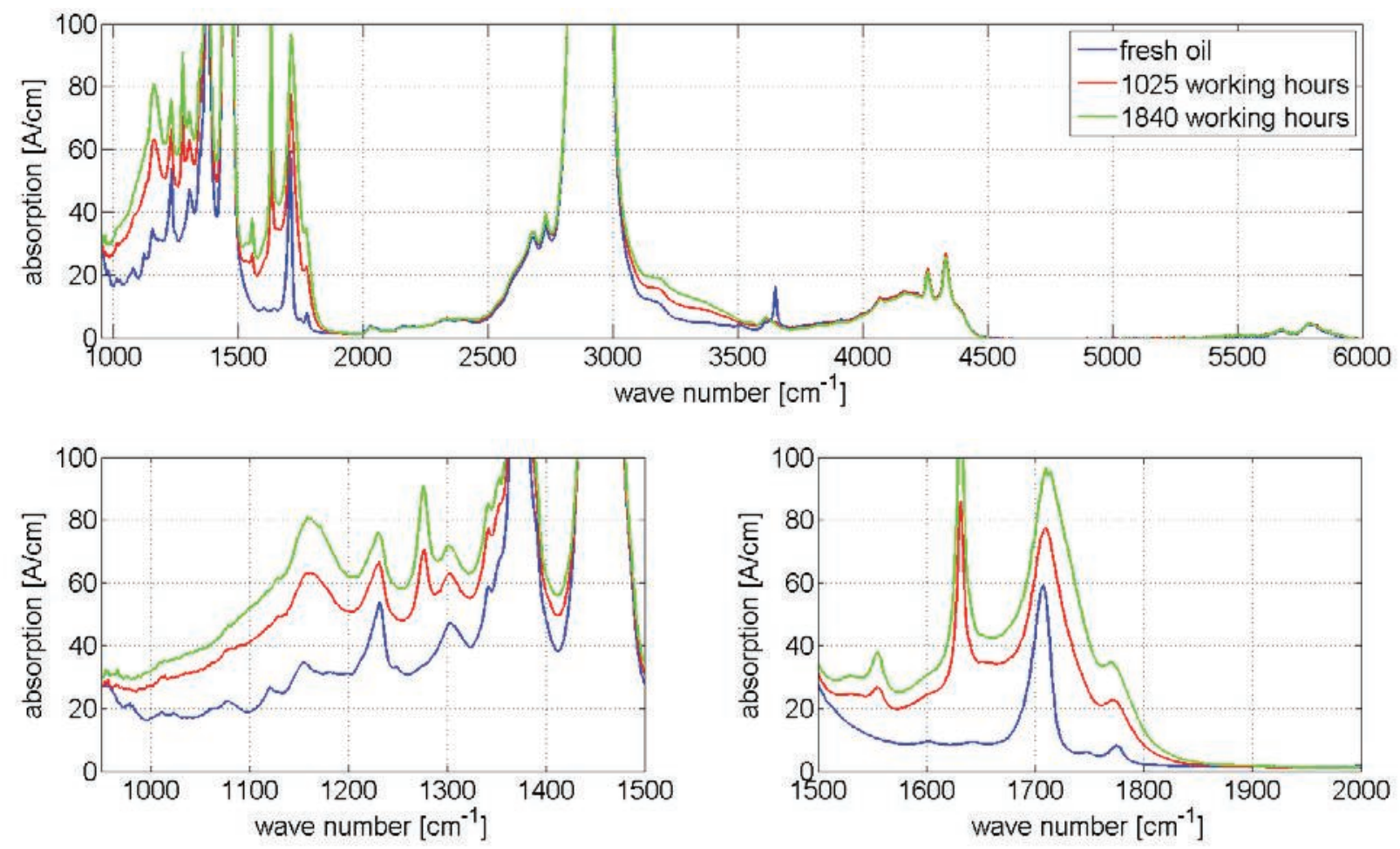

Fig. 6: IR-Spectra of a synthetic oil (Mobil Pegasus 1) from a CHP station for fresh oil, 1025 and 1840 operating hours, respectively. Based on laboratory checks the oil had to be changed after 1840 working hours. Soot content is substracted.

Observing the infrared spectra of the mineral oil, Fig. 5, shows a small increase of the absorption in the wave number range from 3100 to $3700 \mathrm{~cm}^{-1}$, which is also caused by hydroxyl groups. The increased absorption around $1730 \mathrm{~cm}^{-1}$ is due to degradation products of the carbonyl group [8]. For monitoring oxidation and water content of mineral oils the optical filter set of the sensor system was specified at $1720,2500,3350,3500 \mathrm{~cm}^{-1}$.

The artificially aged mineral oil, Fig. 5 , and the synthetic oil of a gas engine from a CHP station, Fig. 6 , show the same characteristic spectral peaks at $1720 \mathrm{~cm}^{-1}$ and around $3300 \mathrm{~cm}^{-1}$. In addition, due to the non artificial aging of the synthetic oil further absorption lines appear which are of interest for true condition monitoring. The increasing absorption at $1630 \mathrm{~cm}^{-1}$ is caused by nitration of the synthetic oil. Around $1150 \mathrm{~cm}^{-1}$ the absorption line of sulfation components is located. Nitration and sulfation in gas engines are the result of the blow by effect when combustion gases like $\mathrm{NO}_{x}$ and $\mathrm{SO}_{x}$ reach the crankcase and react with the oil [10].

For analyzing the artificial and synthetic oil samples the sensor system in the experimental set-up was configured to monitor the oxidation fraction in the spectra. The oxidation and the reference was determined with the filter wavelengths $1720 \mathrm{~cm}^{-1}$ and $2500 \mathrm{~cm}^{-1}$, respectively. The source signal was modulated at $0.1 \mathrm{~Hz}$ with a rectanglular signal and applied continously. The amplified and filtered sensor signals of the synthetic oil are shown in Fig. 7. The detector tuned to the oxidation fraction shows the rectangle signal with the time response of the thermopile sensor. The time constant of each thermopile is approx. $90 \mathrm{~ms}$. Due to increased absorption caused by oxidation the signal shows a decreasing amplitude with increasing oil deterioration. The reference detector signal shows a comparable time behavior as the oxidation detector signal and exhibits only a slight signal decrease caused by aging of the fluid.
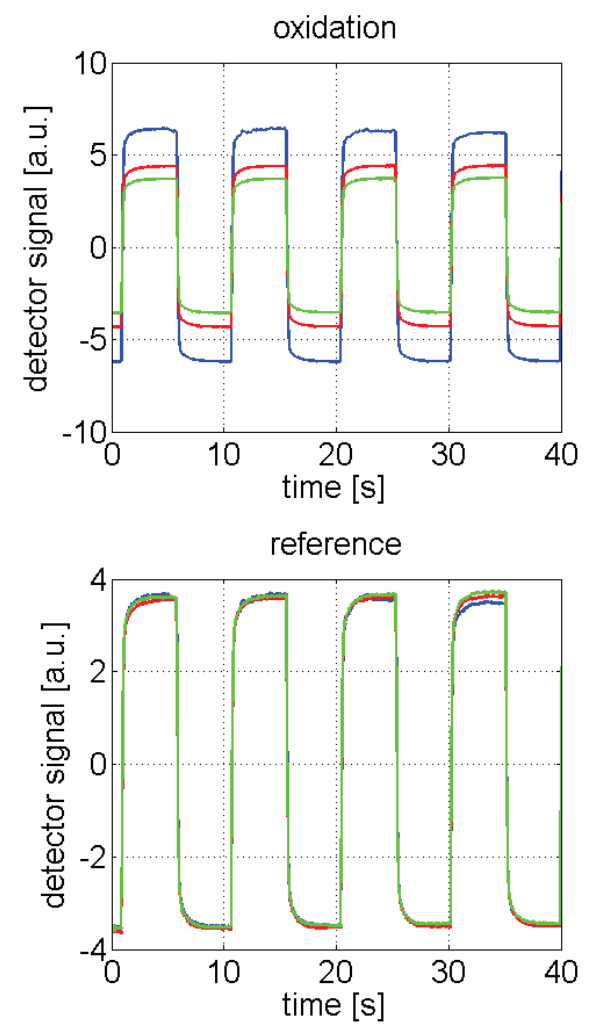

Fig. 7: Raw detector signals for synthetic motor oil (Mobil Pegasus 1). 

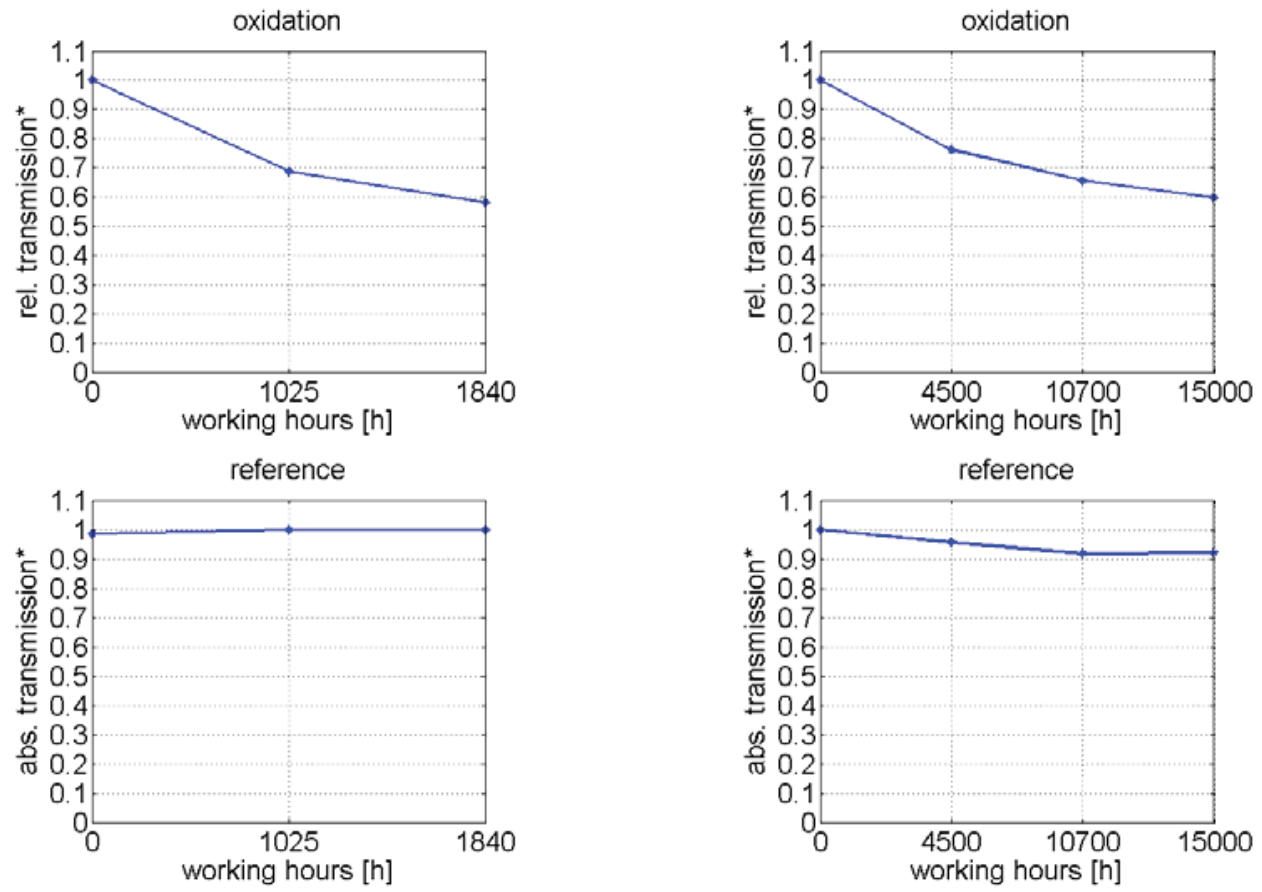

Fig. 8: Normalized detector and reference signals for determining oil deterioration (Mobil Pegasus 1).

Fig. 9: Normalized detector and reference signals for determining oil deterioration (Fuchs HETG).

The sensor signal amplitudes were extracted and used for the histogram analysis to determine the relative transmission of the oil. Fig. 8 shows the relative transmission for the oxidation and the absolute transmission for the reference of the synthetic oil. Here, the oxidation plot was already normalized with the reference signal amplitude to exclude influence of soot and reduced IR intensity. The amplitude of the reference was extracted and normalized to the new oil. The relative transmission of the oxidation shows a strong decrease with increasing deterioration. The reference remains largely unchanged over the whole deterioration of the oil.
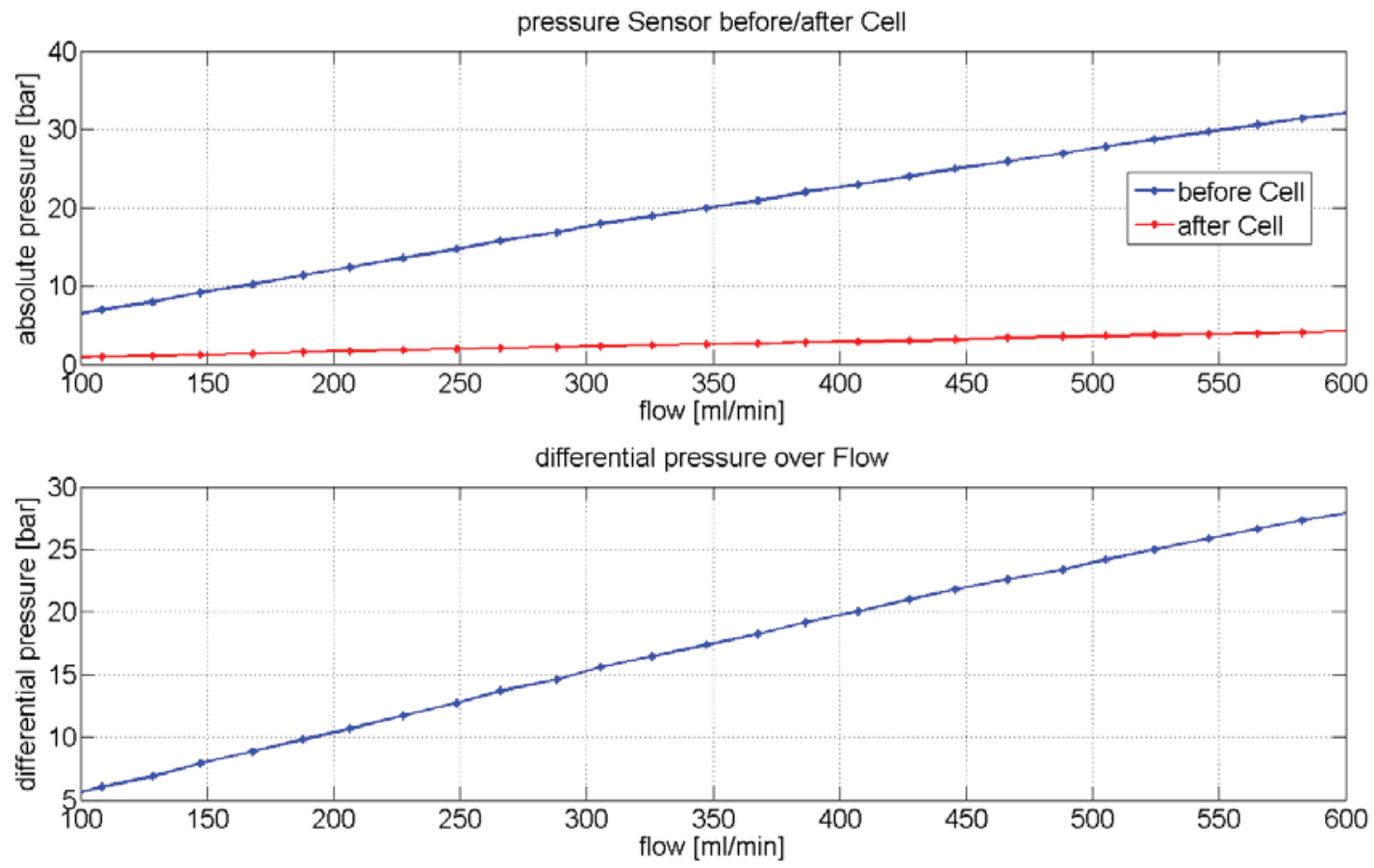

Fig. 10: Differential pressure analyses of the sensor system. Flow rate was changed from 100 to $600 \mathrm{ml} / \mathrm{min}$ using a native oil with a kinematic viscosity of $37 \mathrm{~mm}^{2} / \mathrm{s}$. 
Fig. 9 shows the relative and absolute transmission of the artificially aged native hydraulic oil of Fig. 4 . The absolute transmission of the reference $\left(3875 \mathrm{~cm}^{-1}\right)$ decreases with increasing working hours accordingly to the optical spectrum in Fig. 4 . The working hours were approximated by the reaction rate time rule [11]. For a common hydraulic application temperature was assumed $60^{\circ} \mathrm{C}$ so that an aging temperature of $120^{\circ} \mathrm{C}$ results in a $2^{6}$ faster chemical reaction speed during artificial aging. The relative transmission of the oxidation shows an approximately exponential behavior similar to the synthetic mineral oil in Fig. 8.

Considering the hydraulic properties of the sensor system, the pressure of the sensor system was measured with a swept flow from 100 to $600 \mathrm{ml} / \mathrm{min}$ with native oil with a kinematic viscosity of $37 \mathrm{~mm}^{2} / \mathrm{s}$. The results of the individual pressure sensors placed in front and behind the sensor cell are shown in the upper plot of Fig. 10. The second plot shows the pressure difference obtained by subtracting both sensor signals which corresponds to the pressure loss over the sensor system. The increase of the pressure is approximately linear which indicates a laminar flow of the oil into the measurement cell of the sensor system [12]. By adapting the pressure loss of the sensor system to the desired application, the sensor system can be installed easily in a bypass of the hydraulic system and/or as mobile measurement system for technical fluids for on demand determination of oil aging.

\section{Conclusion and outlook}

A multichannel measurement system for condition monitoring which is based on an optical system using multichannel IR transmission measurements for determining oil deterioration was developed. For evaluating the system native and mineral hydraulic fluids were artificially aged and analyzed in an FTIR spectrometer for comparison. Additional aged samples of synthetic oil from a CHP station were analyzed to compare the results obtained by FTIR spectrometry with the results of the sensor system. Assembled in an experimental set-up and using a simple histogram analysis the sensor system showed a good correlation to the FTIR results and primarily reflected the oxidation of the fluids. In following measurements cross sensitivity effects caused by changes in fluid pressure, flow rate and temperature will be evaluated and improved stimulation signals as well as evaluation methods will be tested.

\section{References}

[1] K. Kudlaty, "Attenuated Total Reflection technique for on-line oil monitoring by means of a FTIR fiber-optic probe", Dissertation, Institute for Measurement Systems and Sensor Technology, TU München, 2004.

[2] C. Schneidhofer et al., „Online-Ölzustandsüberwachung mit chemischen Korrosionssensoren“, Elektrotechnik \& Informationstechnik, 2009, 126/1/2; S. 31-36.

[3] W. Schmidt, "Optische Spektroskopie", Wiley, Vol. 2.

[4] P. Sumit, W. Legner, A. Krenkow, G. Müller, T. Lemettais, F. Pradat, D. Hertens, "Chemical Contamination Sensor for Phosphate Ester Hydraulic Fluids", International Journal of Aerospace Engineering, S. 1-7, 2010.

[5] A. Agoston, C. Schneidhofer, N. Dorr, B. Jakoby; „A concept of an infrared sensor system for oil condition monitoring“, Elektrotechnik \& Informationstechnik, 2008, 125/3, S. 71-75.

[6] M. Fischer et al., „Silicon on ceramics - a new integration concept for silicon devices to LTCC”, Journal of Microelectronics and Electronic Packaging (2009) 6, S. 1-5.

[7] Axetris Leister, "Flyer F60/07", 2008.

[8] ASTM International, "Standard Practice for Condition Monitoring of Used Lubricants by Trend Analysis Using Fourier Transform Infrared (FT-IR)", Spectrometry, E2412 - 04, 2007.

[9] S.K. Naldu, "Evaluation of Liquid Phase Oxidation Products of Ester and Mineral Oil Lubricants“, Ind. Eng. Chem. Prod. Res. Dev.,1984, S. 613-619

[10] Z. Pawlak, "Tribochemistry of Lubricating Oils", Elsevier, Vol. 45

[11] A.F. Holleman, E. Wiberg, "Lehrbuch der Anorganischen Chemie“, deGruyter, Vol. 102, S. 204.

[12] G. Bauer, "Ölhydraulik: Grundlagen, Bauelemente, Anwendungen", Vieweg+ Teubner, Vol. 9, S. 25. 